\title{
Immunodeficiency With Hyper-IgM
}

National Cancer Institute

\section{Source}

National Cancer Institute. Immunodeficiency With Hyper-IgM. NCI Thesaurus. Code C84783.

Immunodeficiency caused by mutations in the gene encoding the CD40 ligand. It is characterized by decreased levels of IgG, IgA, and IgE, and normal or increased levels of IgM. Patients are at an increased risk of infections and development of malignancies. 